\title{
Eficiência agronômica do uso do nitrogênio em genótipos de milho e correlação com caracteres agronômicos
}

\section{Agronomic efficiency of nitrogen use in corn genotypes and correlation with agronomic traits}

\author{
Edmar Vinícius de Carvalho $^{1 *}$, Flávio Sérgio Afférrii ${ }^{1}$, Michel Antônio Dotto ${ }^{1}$, Joênes \\ Mucci Peluzio ${ }^{1}$, Eliane Aparecida Rotili ${ }^{1}$ e Patrícia Bartkow de Almeida ${ }^{1}$ \\ ${ }^{1}$ Departamento de Agronomia; Universidade Federal do Tocantins; 77402-970; Gurupi - TO - Brasil.
}

\begin{abstract}
Looking for to assess the agronomic nitrogen use efficiency (NUE) in different environments and correlation with agronomic characters in corn genotypes, they were evaluated 29 corn genotypes in four experiments, two with seeding organic fertilization. The experiments were deployed in Gurupi-TO on 2010, evaluated these characters: weight hectoliter, weight of 100 grains, grain yield and NUE. The genotypes had shown higher values of NUE under mineral fertilization of seeding without fertilization in coverage, producing $219.9 \mathrm{~kg}_{\mathrm{grain}}^{-1} \mathrm{per} \mathrm{kg}^{-1}$ applied. It's possible to conclude that there is a possibility for indirect selection of genotypes productive and efficient for organic fertilization conditions through choice of efficient genotypes under nitrogen stress conditions.
\end{abstract}

Key-words: Zea mays L., nutritional stress; cattle manure; nitrogen source

\section{INTRODUÇÃO}

O nitrogênio é um dos nutrientes que mais limitam a produção da cultura do milho (Martins et al., 2008), onde a adubação nitrogenada garante o suprimento de alimentos futuramente, devido a resposta de produção com a sua aplicação, em especial na cultura do milho (Liu et al., 2010).

Porém, ao considerar o modelo econômico atual, deve-se levar em consideração o uso racional dos recursos em termos de sustentabilidade ambiental, evitando desperdícios e contaminação do meio ambiente. Dessa forma, a utilização de doses de nitrogênio mais reduzidas que resultam em produção satisfatória pode ser alternativa viável, tendo em vista que a sua adubação em excesso pode causar danos ambientais (Liu et al. 2010).

Prestel et al. (2003) reforçam a ideia da redução da dose nitrogenada, a qual pode ser alcançada por meio do uso de cultivares eficientes quanto ao uso do nitrogênio. Concordando com a ideia de que o melhoramento genético é uma estratégia viável para se aumentar a produtividade por meio do desenvolvimento de genótipos que utilizem de maneira mais eficiente os nutrientes. No entanto, atualmente, os genótipos de milho são desenvolvidos e avaliados em condições de suprimento de nitrogênio, o que pode indicar o lançamento de materiais que não sejam realmente eficientes no uso do nitrogênio (Martins et al., 2008).

Outro ponto de vista é abordado visando à redução de impactos ambientais, a qual foi enfatizada por Kato e Yamagishi (2011), em que a substituição da fonte nitrogenada por resíduos animais em vez de fertilizantes químicos pode ser uma estratégia viável. Estes autores ainda relatam a importância do conhecimento do resíduo animal a ser utilizado, pois a disponibilidade de nitrogênio pode não coincidir com a demanda da planta pelo nutriente, onde a liberação do mesmo pode levar meses a anos de acordo com as condições climáticas. Neste ponto de vista, justifica-se, segundo Annicchiarico 
et al. (2010), o desenvolvimento de programas de melhoramento genético específicos para tal condição. Adicionalmente, ainda existe a possibilidade de se aproveitar parte do programa de melhoramento convencional existente através da seleção indireta.

Assim, o objetivo do presente trabalho foi avaliar a eficiência agronômica do uso do nitrogênio de genótipos de milho em diferentes ambientes e a correlação com caracteres agronômicos.

\section{MATERIAL E MÉTODOS}

Foram instalados quatro experimentos no município de Gurupi - TO (11\%43'45's, 49 04'07' $\mathrm{W})$, em Latossolo vermelho-amarelo distrófico com as seguintes características físicoquímicas: Matéria Orgânica $(\mathrm{MO})=0,4 \% ; \mathrm{pH}$ $(\mathrm{H} 2 \mathrm{O})=5,6 ; \mathrm{P}(\mathrm{Mel})=4,8 \mathrm{mg} \mathrm{L}^{-1} ; \mathrm{K}^{+} ; \mathrm{Ca}^{2+} ; \mathrm{Mg}^{2+}$ e $\mathrm{H}+\mathrm{Al}=0,1 ; 1,2 ; 1,3 ; 3,4 \mathrm{cmol} \mathrm{dm} \mathrm{dm}^{-3}$, respectivamente; $\mathrm{V}=42,2 \%$. Foram avaliados em cada experimento, 29 genótipos de milho, sendo 23 populações experimentais de milho, três híbridos experimentais de milho e três testemunhas. O clima da região é do tipo B1wA'a' úmido com moderada deficiência hídrica, segundo a classificação de Köppen.

A implantação dos experimentos ocorreu no dia 06 de julho de 2010, utilizando-se de irrigação por aspersão, com turno de rega de dois dias, durante todo o ciclo da cultura. Os experimentos foram diferenciados pela combinação entre adubação de semeadura e adubação mineral: dois tipos de adubação na semeadura, mineral $\left(500 \mathrm{~kg} \mathrm{ha}^{-1} \mathrm{da}\right.$ formulação NPK $05-25-15+0,4 \% \mathrm{Zn}-$ denominado de $\mathrm{M})$ e orgânico (40 $\mathrm{t} \mathrm{ha}^{-1}$ de esterco bovino - denominado de Org); e dois níveis de nitrogênio em cobertura, 0 e $125 \mathrm{~kg} \mathrm{ha}{ }^{-1}$, totalizando, quatro experimentos: 1) adubação mineral de semeadura mais $125 \mathrm{~kg} \mathrm{ha}^{-1}$ de $\mathrm{N}$ em cobertura $(125 \mathrm{M})$; 2) adubação orgânica de semeadura mais $125 \mathrm{~kg} \mathrm{ha}^{-1}$ de $\mathrm{N}$ em cobertura (125 Org); 3) adubação mineral de semeadura mais $0 \mathrm{~kg} \mathrm{ha}^{-1}$ de $\mathrm{N}$ em cobertura $(0 \mathrm{M})$ e; 4) adubação orgânica de semeadura mais $0 \mathrm{~kg} \mathrm{ha}^{-1} \mathrm{de}$ $\mathrm{N}$ em cobertura ( 0 Org). $\mathrm{O}$ esterco bovino utilizado na semeadura foi aplicado 15 dias antes no sulco de plantio nos experimentos 125 Org e 0 Org, apresentando as seguintes características: $\mathrm{MO}$ $=10,8 \% ; \mathrm{pH}(\mathrm{H} 2 \mathrm{O})=7,3 ; \mathrm{P}(\mathrm{Mel})=720 \mathrm{mg} \mathrm{L}$; $\mathrm{K}+; \mathrm{Ca} 2+; \mathrm{Mg} 2+\mathrm{e} \mathrm{H}+\mathrm{Al}=24,9 ; 7,9 ; 8,2 ; 2,0$ cmol dm${ }^{-3}$, respectivamente; $\mathrm{V}=91,5 \%$. Nos experimentos que receberam nitrogênio em cobertura (125 M e 125 Org), a adubação foi realizada 34 dias após a semeadura, usando Uréia com fonte nitrogenada.

Em cada experimento, o delineamento experimental foi o de blocos casualizados, com duas repetições, e em cada parcela experimental avaliou-se as duas fileiras centrais de quatro metros de comprimento, com espaçamento $0,9 \mathrm{~m}$ entre linhas. Os tratos culturais usualmente aplicados em cultivo convencional da cultura do milho foram efetuados assim que se fizeram necessários, segundo metodologia de Fancelli e Dourado Neto (2000). A colheita das plantas ocorreu no ponto de colheita de grãos. Foram avaliados o peso hectolítrico, em $\mathrm{kg} 100 \mathrm{~L}^{-1}$; produtividade de grãos, em $\mathrm{kg} \mathrm{ha}^{-1}$, eficiência agronômica do uso do nitrogênio (Moll et al., 1982 - EUN), em $\mathrm{kg}$ grãos ${ }^{-1}$ produzidos por $\mathrm{kg} \mathrm{N}^{-1}$ aplicado e; peso de 100 grãos, em $\mathrm{g}$.

Os dados de EUN, de cada experimento, foram submetidos a uma análise de variância, obedecendo-se ao modelo em blocos casualizados, e posteriormente, os dados de EUN de todos os experimentos, foram submetidos a uma análise de variância conjunta, obedecendo ao critério de homogeneidade dos quadrados médios residuais, sendo realizado, por fim, o teste de médias ScottKnott a $p<0,05$. Foi realizada ainda, Correlação de Pearson utilizando os valores de cada característica biométrica avaliada em cada experimento como fonte de entrada de dados, sendo a significância do coeficiente de correlação dada pelo teste $\mathrm{t}$ a $p<0,05$.

\section{RESULTADOS E DISCUSSÃO}

Houve diferenças significativas $(p<0,01)$ entre genótipos, ambientes (experimentos) e, na interação entre estes dois fatores, com relação a eficiência agronômica do uso do nitrogênio (EUN). Com os genótipos apresentando no experimento $0 \mathrm{M}$ maior EUN $\left(219,9 \mathrm{~kg}\right.$ grãos $\mathrm{kg}^{-1}$ $\mathrm{N})$ que nos demais experimentos (Tabela 1). Com relação a diferença entre os genótipos, verificou-se a formação de dois grupos estatísticos pelo teste de médias Scott-Knott, onde no grupo superior a EUN variou de 86,29 $\mathrm{kg}_{\text {grãos }} \mathrm{kg}^{-1} \mathrm{~N}$ (População

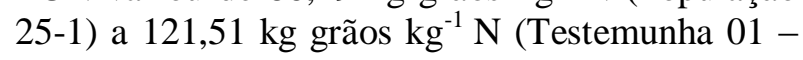
HD). Analisando o resultado dos genótipos no experimento $0 \mathrm{M}$, os mesmos foram divididos em cinco grupos estatísticos pelo teste de médias Scott-Knott, onde no primeiro grupo somente foi classificado a testemunha $01-\mathrm{HD}(383,5 \mathrm{~kg}$ grãos $\left.\mathrm{kg}^{-1} \mathrm{~N}\right)$ e no segundo grupo a população 12-2 (287 $\mathrm{kg}$ grãos $\left.\mathrm{kg}^{-1} \mathrm{~N}\right)$, o híbrido Simples $02(302,7 \mathrm{~kg}$ 
grãos $\left.\mathrm{kg}^{-1} \mathrm{~N}\right)$ e a testemunha 03 - HD $(311,7 \mathrm{~kg}$ grãos $\mathrm{kg}^{-1} \mathrm{~N}$ ). Nos demais experimentos, os genótipos apresentaram EUN similares, ou seja, sem apresentar diferença significativa pelo teste de médias Scott-Knott a $p<0,05$.

A diferença dos genótipos somente no experimento $\mathbf{0 M}$, está de acordo com o encontrado por Prestel et al. (2003), que observaram maior variação genotípica em condições de estresse de nitrogênio. Assim, a escolha de genótipos agronomicamente eficientes quanto o uso de nitrogênio, foi possível apenas nesta condição, a qual pode ser utilizada para a identificação de genitores promissores, pois segundo a afirmação de Martins et al. (2008), genitores ditos como eficientes tendem a gerar híbridos e/ou populações também eficientes.
Com relação aos experimentos que receberam esterco bovino na semeadura (0 Org e 125 Org), a não diferença dos genótipos quanto a EUN nestes experimentos, pode estar ligada a dois fatores: (i) a quantidade de nitrogênio disponibilizada pelo esterco bovino no primeiro cultivo é diferente da quantidade contida no mesmo (a qual foi utilizada no cálculo da eficiência), pois, segundo Kato e Yamagishi (2011) em adubos orgânicos especificamente esterco bovino) a disponibilidade de nitrogênio para as plantas é influenciada pelas condições climáticas da região; e (ii), os benefícios da adubação orgânica nas propriedades físicoquímicas do solo, onde Liu et al. (2010) ressaltam que a ação do adubo orgânico no solo pode ser a razão da produtividade de grãos de milho semelhante a condição de adubação mineral.

Tabela 1. Eficiência agronômica do uso do nitrogênio segundo Moll et al. (1982) de 29 genótipos de milho semeados em quatro experimentos, na entressafra 2010 em Gurupi-TO.

\begin{tabular}{|c|c|c|c|c|c|c|c|c|}
\hline \multirow{2}{*}{ Genótipos } & \multicolumn{6}{|c|}{ Experimentos } & \multirow{2}{*}{\multicolumn{2}{|c|}{ Média }} \\
\hline & 0 Org & $0 \mathrm{M}$ & & 1250 & & $125 \mathrm{M}$ & & \\
\hline $1-3$ & 27,90 & 169,0 & $\mathrm{E}$ & 21,09 & & 46,25 & 66,07 & B \\
\hline $1-5$ & 25,07 & 192,7 & $\mathrm{E}$ & 17,10 & & 27,26 & 65,54 & B \\
\hline $10-1 / 1$ & 20,51 & 200,7 & D & 12,62 & & 30,21 & 66,02 & B \\
\hline $10-1 / 2$ & 31,30 & 225,4 & D & 19,97 & & 32,97 & 77,41 & B \\
\hline $10-6 / 1$ & 19,84 & 140,8 & $\mathrm{E}$ & 15,34 & & 24,15 & 50,03 & B \\
\hline $10-6 / 2$ & 17,55 & 154,1 & $\mathrm{E}$ & 12,41 & & 12,69 & 49,19 & B \\
\hline $11-3$ & 29,25 & 237,7 & D & 19,69 & & 39,04 & 81,42 & B \\
\hline $12-2$ & 27,30 & 287,0 & B & 23,04 & & 36,18 & 93,39 & A \\
\hline $12-3$ & 27,24 & 208,5 & D & 21,13 & & 47,74 & 76,16 & B \\
\hline $12-5$ & 26,68 & 226,7 & $\mathrm{D}$ & 18,03 & & 32,53 & 75,99 & B \\
\hline $12-6$ & 23,33 & 190,9 & $\mathrm{E}$ & 18,45 & & 27,89 & 65,14 & B \\
\hline $15-1 / 1$ & 30,02 & 247,5 & $\mathrm{C}$ & 26,76 & & 46,27 & 87,65 & A \\
\hline $15-1 / 2$ & 25,80 & 165,9 & $\mathrm{E}$ & 20,15 & & 32,84 & 61,18 & B \\
\hline $15-3 / 1$ & 14,04 & 211,7 & $\mathrm{D}$ & 19,02 & & 21,92 & 66,68 & B \\
\hline $15-3 / 2$ & 22,59 & 171,1 & $\mathrm{E}$ & 16,63 & & 24,44 & 58,70 & B \\
\hline $25-1$ & 30,31 & 268,7 & $\mathrm{C}$ & 21,46 & & 24,74 & 86,29 & A \\
\hline $25-2$ & 24,41 & 185,9 & $\mathrm{E}$ & 23,33 & & 31,83 & 66,36 & B \\
\hline $25-5$ & 19,86 & 214,1 & $\mathrm{D}$ & 17,35 & & 27,86 & 69,79 & B \\
\hline $26-1$ & 33,57 & 224,6 & $\mathrm{D}$ & 19,07 & & 32,51 & 77,45 & B \\
\hline $26-2$ & 17,53 & 210,9 & $\mathrm{D}$ & 19,12 & & 36,18 & 70,93 & B \\
\hline $28-5$ & 28,20 & 157,8 & $\mathrm{E}$ & 19,80 & & 37,68 & 60,87 & B \\
\hline $35-5$ & 24,18 & 183,8 & $\mathrm{E}$ & 19,02 & & 36,93 & 65,99 & B \\
\hline Híbrido Simples 01 & 34,06 & 153,9 & $\mathrm{E}$ & 23,36 & & 43,84 & 63,79 & B \\
\hline Híbrido Triplo 01 & 36,68 & 264,6 & $\mathrm{C}$ & 26,11 & & 59,54 & 96,74 & A \\
\hline Híbrido Simples 02 & 39,16 & 302,7 & B & 30,47 & & 41,52 & 103,46 & A \\
\hline Testemunha 01 - HD & 47,43 & 383,5 & A & 27,60 & & 27,54 & 121,51 & A \\
\hline Testemunha 02 - Variedade & 26,27 & 236,4 & $\mathrm{D}$ & 19,03 & & 30,15 & 77,96 & B \\
\hline Testemunha 03 - HD & 30,46 & 311,7 & B & 11,81 & & 49,84 & 100,96 & A \\
\hline P.O & 34,40 & 248,5 & $\mathrm{C}$ & 25,80 & & 38,81 & 86,88 & A \\
\hline Média & $27,41 \mathrm{~b}$ & 219,9 & $\mathrm{a}$ & 20,16 & $\mathrm{c}$ & 34,53 & b 75,50 & \\
\hline
\end{tabular}




\begin{tabular}{cccc}
\hline CV (\%) & & & 29,73 \\
Significância & Genótipos & Ambientes & Interação \\
Teste F & $1 \%$ & $1 \%$ & $1 \%$ \\
\hline
\end{tabular}

Médias seguidas de mesmas letras maiúsculas na coluna e minúsculas na linha não apresentam diferença significativa pelo teste Scott-Knott à 5\%. $\mathbf{0} \mathbf{M}=$ adubação de semeadura mineral sem nitrogênio em cobertura; $125 \mathbf{M}=$ adubação de semeadura mineral com nitrogênio em cobertura; 0 Org = adubação orgânica de semeadura sem nitrogênio em cobertura; 125 Org = adubação orgânica de semeadura com nitrogênio em cobertura.

Todos os coeficientes de correlação entre o peso hectolítrico $(\mathrm{PH})$ dos genótipos avaliados com a produtividade de grãos (PROD) e EUN que foram significativos, apresentaram correlação negativa (Tabela 2). Ao contrário do PH, os coeficientes de correlação entre peso 100 grãos (P) com a PROD e a EUN que foram significativos, apresentando valores positivos. Resultados semelhantes também foram encontrados por Cancellier et al. (2011), em populações tropicais de milho avaliadas na mesma região do presente trabalho, em anos anteriores.

Tabela 2. Coeficientes da Correlação de Pearson entre as características avaliadas em cada experimento e a produtividade de grãos (PR) e a eficiência agronômica do uso do nitrogênio (EF) segundo Moll et al. (1982), na entre-safra 2010, Gurupi-TO.

\begin{tabular}{|c|c|c|c|c|c|c|c|c|c|c|}
\hline & $\mathrm{PR}^{3}$ & $\mathrm{PR}^{1}$ & $\mathrm{PR}^{4}$ & $\mathrm{PR}^{2}$ & $\mathrm{PR}^{\mathrm{M}}$ & $\mathrm{EF}^{3}$ & $\mathrm{EF}^{1}$ & $\mathrm{EF}^{4}$ & $\mathrm{EF}^{2}$ & $\mathrm{EF}^{\mathrm{M}}$ \\
\hline $\mathbf{P H}^{\mathbf{3}}$ & $-0,21$ & $-0,16$ & $-0,29$ & $-0,12$ & $-0,25$ & $-0,20$ & $-0,16$ & $-0,29$ & $-0,11$ & $-0,19$ \\
\hline $\mathbf{P H}^{1}$ & $-0,16$ & $-0,07$ & $-0,28$ & $-0,03$ & $-0,17$ & $-0,15$ & $-0,07$ & $-0,29$ & $-0,02$ & $-0,10$ \\
\hline $\mathbf{P H}^{4}$ & $-0,25$ & $-0,26$ & $-0,13$ & $-0,01$ & $-0,21$ & $-0,24$ & $-0,26$ & $-0,15$ & $-0,01$ & $-0,26$ \\
\hline $\mathbf{P H}^{2}$ & $-0,47^{* *}$ & $-0,53^{* *}$ & $-0,51^{* *}$ & $-0,14$ & $-0,52^{* *}$ & $-0,46^{*}$ & $-0,53^{* *}$ & $-0,50^{* *}$ & $-0,14$ & $-0,55^{* *}$ \\
\hline $\mathbf{P H}^{\mathbf{M}}$ & $-0,42^{*}$ & $-0,38^{*}$ & $-0,48^{* *}$ & $-0,11$ & $-0,44^{*}$ & $-0,41^{*}$ & $-0,38^{*}$ & $-0,49^{* *}$ & $-0,11$ & $-0,41^{*}$ \\
\hline $\mathbf{P}^{3}$ & $0,55^{* *}$ & 0,31 & $0,43^{*}$ & $0,50^{* *}$ & $0,57^{* *}$ & $0,56^{* *}$ & 0,31 & $0,43^{*}$ & $0,51^{* *}$ & $0,46^{*}$ \\
\hline $\mathbf{P}^{1}$ & $0,40^{*}$ & $0,37^{*}$ & 0,25 & $-0,03$ & 0,32 & $0,40^{*}$ & $0,37^{*}$ & 0,24 & 0,00 & $0,36^{*}$ \\
\hline $\mathbf{P}^{4}$ & $0,41^{*}$ & 0,33 & $0,38^{*}$ & $0,38^{*}$ & $0,48^{* *}$ & $0,40^{*}$ & 0,33 & $0,40^{*}$ & $0,38^{*}$ & $0,41^{*}$ \\
\hline $\mathbf{P}^{2}$ & $0,37^{*}$ & $0,41^{*}$ & $0,38^{*}$ & $0,53^{* *}$ & $0,53^{* *}$ & 0,36 & $0,41^{*}$ & $0,38^{*}$ & $0,53^{* *}$ & $0,49^{* *}$ \\
\hline $\mathbf{P}^{\mathrm{M}}$ & $0,61^{* *}$ & $0,49^{* *}$ & $0,52^{* *}$ & $0,49^{* *}$ & $0,67^{* *}$ & $0,61^{* *}$ & $0,49^{* *}$ & $0,52^{* *}$ & $0,50^{* *}$ & $0,59^{* *}$ \\
\hline $\mathbf{P R}^{3}$ & - & $0,66^{* *}$ & $0,69^{* *}$ & $0,48^{* *}$ & $0,89^{* *}$ & $0,99^{* *}$ & $0,66^{* *}$ & $0,70^{* *}$ & $0,49^{* *}$ & $0,77^{* *}$ \\
\hline $\mathbf{P R}^{1}$ & - & - & $0,46^{*}$ & 0,25 & $0,74^{* *}$ & $0,65^{* *}$ & $1,00^{* *}$ & $0,48^{* *}$ & 0,26 & $0,97^{* *}$ \\
\hline $\mathbf{P R}^{4}$ & - & - & - & $0,43^{*}$ & $0,82^{* *}$ & $0,68^{* *}$ & $0,46^{*}$ & $0,99^{* *}$ & $0,44^{*}$ & $0,59^{* *}$ \\
\hline $\mathbf{P R}^{2}$ & - & - & - & - & $0,69^{* *}$ & $0,48^{* *}$ & 0,26 & $0,44^{*}$ & $0,99^{* *}$ & $0,44^{*}$ \\
\hline $\mathbf{P R}^{\mathrm{M}}$ & - & - & - & - & - & $0,89^{* *}$ & $0,74^{* *}$ & $0,83^{* *}$ & $0,70^{* *}$ & $0,87^{* *}$ \\
\hline $\mathbf{E F}^{3}$ & - & - & - & - & - & - & $0,65^{* *}$ & $0,70^{* *}$ & $0,49^{* *}$ & $0,77^{* *}$ \\
\hline $\mathbf{E F}^{1}$ & - & - & - & - & - & - & - & $0,48^{* *}$ & 0,26 & $0,97^{* *}$ \\
\hline $\mathbf{E F}^{4}$ & - & - & - & - & - & - & - & - & $0,45^{*}$ & $0,61^{* *}$ \\
\hline $\mathbf{E F}^{2}$ & - & - & - & - & - & - & - & - & - & $0,45^{*}$ \\
\hline
\end{tabular}

PH = Peso hectolítrico. $\mathrm{P}=$ peso de 100 grãos; ${ }^{1}$ adubação de semeadura mineral sem nitrogênio em cobertura; ${ }^{2}$ adubação de semeadura mineral com nitrogênio em cobertura; ${ }^{3}$ adubação orgânica de semeadura sem nitrogênio em cobertura; ${ }^{4}$ adubação orgânica de semeadura com nitrogênio em cobertura; ${ }^{\mathrm{M}}$ média dos quatro experimentos. ${ }^{* * *}$ Significância a $5 \%$ e $1 \%$ pelo teste $\mathrm{t}$. 
Não foram encontradas diferenças significativas da correlação entre a PROD nos experimentos com adubação de semeadura mineral $(r=0,25)$. Comportamento semelhante foi observado para EUN $(r=0,26)$. Esse fato pode estar ligado ao encontrado por Prestel et al. (2003), onde os autores observaram decréscimo do coeficiente de correlação entre a produtividade de grãos de milho em alto e baixo nitrogênio, quando a condição de baixo nitrogênio atingia níveis de deficiência e estresse. Assim, como houve correlação entre a PROD no experimento $125 \mathrm{M}$ com a PROD no experimento $0 \operatorname{Org}\left(r=0,48^{*}\right)$, pode-se sugerir que mesmo não recebendo nitrogênio em cobertura no experimento 0 Org, a adubação orgânica de semeadura, de certa forma, foi capaz de suprir nitrogênio aos genótipos semelhante ao fornecido via adubação mineral (semeadura e cobertura), logo num primeiro cultivo.

A PROD média apresentou coeficientes de correlação significativos com a EUN dos genótipos de cada experimento (variando de $\mathrm{r}=$ $0,70^{* *}$ a $\left.\mathrm{r}=0,89^{* *}\right)$. E entre os resultados de EUN, o coeficiente de correlação entre a EUN no experimento $0 \mathrm{M}$ e a EUN média foi significativo $\left(\mathrm{r}=0,97^{* *}\right)$, sendo maior que dos demais coeficientes. O coeficiente de correlação entre EUN no experimento $0 \mathrm{M}$ e a PROD no experimento 0 Org $\left(r=0,66^{* *}\right)$ dos genótipos avaliados, indicou que a seleção de genótipos eficientes em condições de estresse de nitrogênio, indiretamente, em partes, pode promover a escolha de genótipos produtivos em condições de adubação orgânica (40 t ha ${ }^{-1}$ de esterco bovino) sem adubação nitrogenada de cobertura.

Lorenzana e Bernardo (2008) encontraram na produção de grãos a possibilidade de selecionar genótipos promissores em condições orgânicas, por meio de seleção indireta em condições convencionais. A qual pode ser eficiente quando há correlação entre os ambientes juntamente com maior variabilidade no ambiente em que se deseja fazer a seleção indireta (Lorenzana e Bernardo 2008). Sendo encontrado este fato quanto a EUN no presente trabalho, onde no experimento $(0 \mathrm{M})$ foi possível a visualização da diferenças entre os genótipos quanto a eficiência agronômica do uso do nitrogênio (Tabela 1), e houve correlação da EUN neste experimento com a EUN e PROD nos experimentos com adubação orgânica de semeadura (Tabela 2). Assim, provavelmente existe a possibilidade de inicialmente os programas de melhoramento visando esta característica voltada ao cultivo orgânico, serem desenvolvidos em ambientes de estresse de nitrogênio, para a seleção prévia dos genótipos eficientes, e posteriormente, levados a avaliações nas condições as quais serão cultivados a campo.

\section{CONCLUSÕES}

$\mathrm{O}$ ambiente de maior estresse nutricional (de nitrogênio) foi o mais adequado na distinção dos genótipos quanto à eficiência agronômica do uso do nitrogênio. Existe a possibilidade de seleção indireta de genótipos produtivos e eficientes para condições de adubação orgânica por meio de condições em estresse de nitrogênio.

\section{AGRADECIMENTOS}

À Coordenação de Aperfeiçoamento de Pessoal do Nível Superior (CAPES) pela bolsa de estudos do autor correspondente. E ao Conselho Nacional de Desenvolvimento Científico e Tecnológico (CNPQ) pelo financiamento do projeto.

\section{RESUMO}

Buscando avaliar a eficiência agronômica do uso do nitrogênio (EUN) em diferentes ambientes e a correlação com características agronômicas em genótipos de milho, foram avaliados 29 genótipos em quatro experimentos, sendo dois com adubação orgânica de semeadura. Os experimentos foram conduzidos em Gurupi-TO em 2010, avaliando-se peso hectolitro, peso de 100 grãos, produtividade de grãos e EUN. Os genótipos apresentaram maiores valores de EUN com adubação mineral de semeadura sem adubação em cobertura, produzindo $219,9 \mathrm{~kg}_{\text {grão }}{ }^{-1}$ por $\mathrm{kg} \mathrm{N} \mathrm{N}^{-1}$ aplicado. A seleção indireta de genótipos produtivos e eficientes para condições de adubação orgânica por meio de condições em estresse de nitrogênio é viável e pode ser utilizada para diferentes genótipos de Zea mays.

Palavras-chave: Zea mays L., estresse nutricional, esterco bovino, fonte de nitrogênio

\section{REFERENCIAS}

Annicchiarico, P.; Chiapparino, E.; Perezin, M. (2010), Response of common wheat varieties to organic and conventional production systems across Italian locations, and implications for selection. Field Crops Research, 116, 230-238.

Cancellier, L. L.; Afférri, F. S.; Carvalho, E. V.; Dotto, M. A.; Leão, F. F. (2011), Eficiência no uso de nitrogênio e correlação fenotípica em populações tropicais de milho no Tocantins. Revista Ciência Agronômica, 42, 139-148. 
Fancelli, A. L. e Dourado Neto, D. (2000), Produção de milho. Guaíba: Agropecuária, 360p.

Kato, Y. e Yamagishi, J. (2011), Long-term effects of organic manure application on the productivity of winter wheat grown in a crop rotation with maize in Japan. Field Crops Research, 120, 387395.

Liu, J.; Liu, H.; Huang, S.; Yang, X.; Wang, B.; Li, X.; Ma, Y. (2010), Nitrogen efficiency in longterm wheat-maize cropping systems under diverse field sites in China. Field Crops Research, 118, 145-151.

Lorenzana, R. E. e Bernado, R. (2008), Genetic Correlation between Corn Performance in Organic and Conventional Production Systems. Crop Science, 48, 903-910.
Martins, A. O.; Campostrini, E.; Magalhães, P. C.; Guimarães, L. J. M.; Durães, F. O. M.; Marriel, I. E.; Torres Neto, A. (2008), Nitrogen-use efficiency of maize genotypes in contrasting environments. Crop Breeding and Applied Biotechnology, 8, 291-298.

Moll, R. H.; Kamprath, E. J.; Jackson, W. A, (1982), Analysis and interpretation of factors which contribute to efficiency of nitrogen utilization. Agronomy Journal, 74, 562-564.

Prestel, T.; Seitz, G.; Landbeck, M.; Thiemt, E. M.; Schmidt, W.; Geiger, H. H. Improving Nitrogen-Use Efficiency in European Maize: Estimation of Quantitative Genetic Parameters. Crop Science, 43, 1259-1265. 